J. Med. Microbiol. - Vol. 26 (1988), 87-91

(C) 1988 The Pathological Society of Great Britain and Ireland

\title{
Enterotoxigenicity of chicken isolates of Campylobacter jejuni in ligated ileal loops of rats
}

\author{
S. K. SAHA, N. P. SINGH and S. C. SANYAL*
}

\begin{abstract}
Enteric Infection Unit, Department of Microbiology, Institute of Medical Sciences, Banaras Hindu University, Varanasi 221 005, India
\end{abstract}

Summary. Ligated ileal loops in rats of the Charles-Foster strain, weighing $200-250 \mathrm{~g}$ and aged 7-8 months, provided a sensitive and reproducible means of testing the enterotoxigenicity of Campylobacter jejuni. All of 16 chicken isolates caused fluid accumulation comparable to that produced by toxigenic Vibrio cholerae 01 , strain 569B. However, 11 of the isolates required one to three consecutive passages through the rat gut before doing so. The amount of fluid produced increased after each passage. Of three culture media tested, brucella broth of $p \mathrm{H} 6.7$ supported the highest degree of enterotoxigenesis. Filtrates of cultures of all 16 chicken isolates in this medium were as effective as viable $C$. jejuni in producing fluid in ileal loops. The enterotoxin was neutralised completely by cholera antitoxin diluted 1 in 160 , indicating its close immunobiological relationship to cholera toxin.

\section{Introduction}

Campylobacter jejuni is one of the commonest causes of diarrhoea in many countries (Walker $e t$ al., 1986) and has also been implicated as a cause of traveller's diarrhoea (Speelman et al., 1983). Some strains from patients with diarrhoea produce heat-labile enterotoxin (LT) (Ruiz-Palacios et al., 1983; Johnson and Lior, 1984; McCardell et al., 1984). Partial antigenic and other similarities between this enterotoxin and the LT of both Vibrio cholerae $\mathrm{O} 1$ and Escherichia coli have also been demonstrated (Ruiz-Palacios et al., 1983; Klipstein and Engert, 1984, 1985; McCardell et al., 1984). Chickens were found to be the commonest reservoir of $C$. jejuni (Harris et al., 1986), but there is no published information on the enterotoxigenicity of chicken isolates. The objectives of the present study were to determine whether (1) chicken isolates cause fluid accumulation in ligated ileal loops of rats, (2) consecutive passages through the rat gut enhance the enterotoxigenicity of non- or weakly toxigenic strains, and (3) the enterotoxin of chicken isolates is neutralised by cholera antitoxin.

\section{Materials and methods}

\section{Organisms}

Sixteen strains of $C$. jejuni biotype 2 isolated from the intestines of broiler chickens from different sources were

Received 21 Sep. 1987; accepted 18 Nov. 1987.

* Correspondence should be sent to Professor S. C. Sanyal. identified by standard tests (Smibert, 1978; Skirrow and Benjamin, 1980) and preserved in a stock medium of nutrient broth containing glycerol $10 \%$ and FBP (ferrous sulphate, sodium bisulphite and sodium pyruvate) supplement $0.025 \%$ at $-90^{\circ} \mathrm{C}$ (George et al., 1978), each strain being distributed into five tubes. None of the strains had undergone more than two subcultures before being tested for enterotoxin production. Vibrio cholerae strain 569B maintained in our laboratory served as a positive control in initial experiments.

\section{Media}

Preliminary tests were made with various modifications of Brucella Broth (Gibco) to find the best medium for toxin production. The modifications were (1) the adjustment of $p \mathrm{H}$ to different values between 5 and 10 , (2) addition of $\mathrm{L}$-asparagine $0.25 \%$, L-serine $0.25 \%$ and $\mathrm{L}$ glutamic acid $0 \cdot 25 \%$, and (3) addition of polymyxin B, $4 \mathrm{IU} / \mathrm{ml}$ (Ruiz-Palacios et al., 1983). Brucella broth alone at $p \mathrm{H} 6.7$ supported the maximum degree of toxigenesis and of growth as judged by optical density. No significant increase in the yield of toxin was observed on the addition of polymyxin $\mathrm{B}$ or on the readjustment of $p \mathrm{H}$ to 6.7 after amino acid supplementation.

\section{Preparation of culture filtrates}

Five or six colonies of each $C$. jejuni strain grown for $48 \mathrm{~h}$ in a candle jar at $42^{\circ} \mathrm{C}$ on Campy-BAP (Hi-Media) medium with antibiotic supplement (Blaser et al., 1979) were inoculated into $10 \mathrm{ml}$ of Brucella broth, $p \mathrm{H} \mathrm{6.7 \text {, }}$ contained in $50-\mathrm{ml}$ conical flasks. The cultures were incubated for $72 \mathrm{~h}$ in a candle jar without shaking. After 
centrifugation, the culture supernates were filtered through millipore membranes of $0 \cdot 22-\mu \mathrm{m}$ average pore diameter, dispensed in small volumes and stored at $4^{\circ} \mathrm{C}$ (Ruiz-Palacios et al., 1983). Culture filtrate was prepared only when live cells of a strain caused fluid accumulation in the rat ileal loop either before or after passage through the gut of rats. Culture filtrate of the positive control strain $V$. cholerae 569B was prepared in Richardson's medium by a method described earlier (Sanyal et al., 1984).

\section{Ileal loop tests in adult rabbits}

Live cells of the $16 C$. jejuni isolates were tested in New Zealand albino rabbits by the method of Annapurna and Sanyal (1977). The strains, grown in Brucella broth for $18 \mathrm{~h}$, were injected into rabbit ileal loops in doses of $1.0 \mathrm{ml}$ containing $10^{6-7} \mathrm{cfu}$. The known toxigenic $V$. cholerae strain 569B grown in Brain Heart Infusion Broth (Difco), and unseeded Brucella broth, served as positive and negative controls respectively. Culture filtrates of all the strains prepared in Brucella broth of $p \mathrm{H} 6.7$ were tested in a similar way in 1-ml doses. Each test was done in triplicate.

\section{Ileal loop tests in rats (Charles-Foster strain)}

Albino rats weighing 100-250 g were used to examine the production of enterotoxin by $C$. jejuni strains. Faecal specimens of the rats were cultured for $C$. jejuni for three consecutive days before the tests, and all yielded negative results. The rats were starved for $48 \mathrm{~h}$ but given water $a d$ libitum. The intestines of each rat were exposed by abdominal incision under light ether anaesthesia and 68 loops of $5 \mathrm{~cm}$ length at $2 \mathrm{~cm}$ intervals were tied, starting from the ileo-caecal junction. Live cells and culture filtrates were injected into these loops as in the rabbit gut loop tests (above), except that the dose was $0.5 \mathrm{ml}$. $V$. cholerae strain 569B and Brucella broth served as positive and negative control material respectively. The rats were killed after $18 \mathrm{~h}$ and the length and fluid content of each loop were measured. Each experiment was done in triplicate.

Consistent and reproducible results with both live cells and culture filtrates were obtained in rats weighing 200 $250 \mathrm{~g}$ and aged 7-8 months; rats weighing less than $200 \mathrm{~g}$ gave inconsistent results. Live cells or culture filtrates of C. jejuni strain 9 consistently caused fluid accumulation in the range of $0.5-0.7 \mathrm{ml} / \mathrm{cm}$ of gut in 10 initial experiments. In subsequent experiments, therefore, it replaced $V$. cholerae 569B as the positive control strain.

\section{Passage in rat ileal loops}

Consecutive passages of the $C$. jejuni strains that caused little or no accumulation of fluid in initial experiments were made in rat gut loops as described earlier (Shankar et al., 1982; Sanyal et al., 1984). In short, such strains were subcultured from the gut loops on Campy-BAP plates and incubated in a candle jar at $42^{\circ} \mathrm{C}$ for $48 \mathrm{~h}$. Five to six colonies of each strain were grown in Brucella broth at $42^{\circ} \mathrm{C}$ for $18 \mathrm{~h}$ in a candle jar and $0.5 \mathrm{ml}$ volumes were inoculated again into rat loops. The process was continued until good positive responses were obtained.

\section{Determination of the optimum loop-reacting dose}

Cell-free culture filtrates of the $C$. jejuni strains numbered 2, 5, 9, 10 and 13 were injected in volumes ranging between 0.1 and $0.6 \mathrm{ml}$ into rat ileal loops to find the dose causing a maximal reaction (Annapurna and Sanyal, 1977). Filtrates in doses of $0.25 \mathrm{ml}$ caused fluid accumulations of about $0.5 \mathrm{ml} / \mathrm{cm}$ of gut. This amount of fluid was no greater when larger volumes of culture filtrate were injected (fig. 1). The $0.25 \mathrm{ml}$ dose of filtrate was used in neutralisation tests with cholera antitoxin in rat loops.

\section{Neutralisation of $C$. jejuni enterotoxin by cholera antitoxin in rat ileal loops}

Five strains $(2,5,9,10$ and 13$)$ were tested in rat loops by a method used earlier in rabbits (Dubey and Sanyal, 1978; Sanyal et al., 1984). Culture filtrate $(0.25 \mathrm{ml})$ was mixed with $0.25 \mathrm{ml}$ of dilutions of anti-CT (obtained from NIH, USA) in PBS of $p \mathrm{H} 7.2$ as described earlier (Dubey and Sanyal, 1978; Lahiri et al., 1982; Sanyal et al., 1984). The mixtures were incubated at $37^{\circ} \mathrm{C}$ in a water bath for $30 \mathrm{~min}$ and then tested in rat ileal loops to find the highest dilution of cholera antitoxin that would completely neutralise enterotoxic activity. Culture filtrate $(0.25 \mathrm{ml})$ mixed with an equal volume of PBS served as a positive control inoculum.

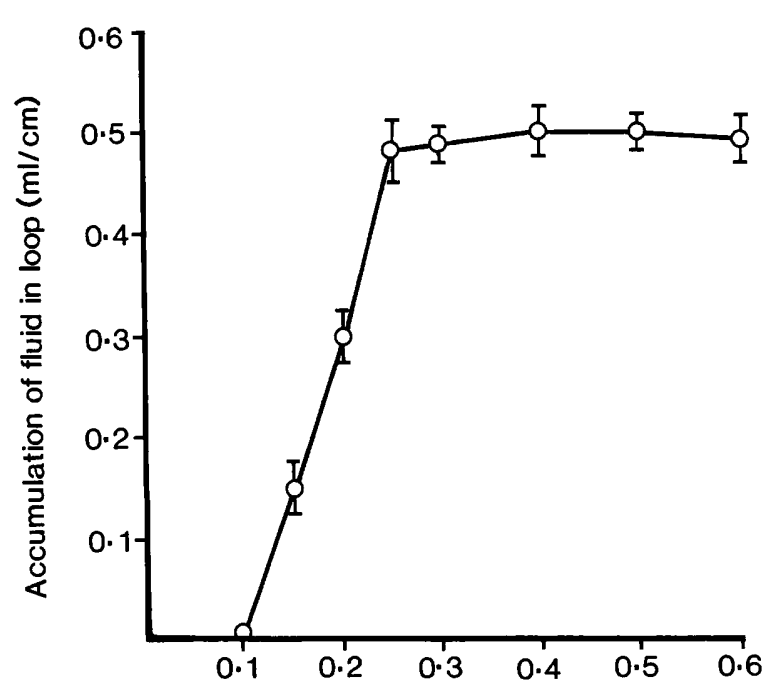

Amount of culture filtrate injected into loops $(\mathrm{ml})$

Fig. 1. Determination of optimum loop reacting doseaccumulation of fluid with different doses of culture filtrates. The results are the means $( \pm S D)$ for filtrates of five cultures. 


\section{Results}

\section{Ileal loop tests}

In rabbit gut loops, neither live cells nor culture filtrate of any $C$. jejuni strain caused fluid accumulation, but mild to severe haemorrhage was sometimes observed. $V$. cholerae strain no. 569B and Brucella broth invariably gave positive and negative reactions respectively.

In initial experiments with rat ileal loops, live cells of only five of the $16 C$. jejuni strains caused fluid accumulation comparable with that of the known toxigenic strain (569B) of $V$. cholerae. Of the 11 strains that gave negative results initially, four became positive after a single passage through rat gut and four and three after two and three passages, respectively. Fluid production was enhanced by each consecutive passage and after the third the volume was $\geqslant 0.5 \mathrm{ml} / \mathrm{cm}$ of gut. Filtrates caused accumulations of fluid in the range from $0.25-0.7 \mathrm{ml}$ (table), i.e., volumes similar to those produced by live cells.

\section{Neutralisation of $C$. jejuni enterotoxin by cholera antitoxin}

The highest dilution that completely neutralised the enterotoxic activity of culture filtrate $(0 \cdot 25 \mathrm{ml})$ of strains $2,5,9,10$ and 13 was 1 in 160 (fig. 2). There was proportionately less neutralisation (i.e., more fluid accumulation) with higher serial dilutions of cholera antitoxin (fig. 3).

Table. Enhancement of the fluid-producing effect of $C$. jejuni culture filtrates by passage through rat ileal loops

\begin{tabular}{ccc}
\hline & \multicolumn{2}{c}{ Fluid produced $(\mathrm{ml} / \mathrm{cm}$ of gut $)$} \\
\cline { 2 - 3 } Strain & before passage & after passage \\
\hline 1 & 0 & $0 \cdot 30-0 \cdot 50$ \\
2 & 0 & $0 \cdot 44-0 \cdot 55$ \\
3 & 0 & $0 \cdot 20-0 \cdot 30$ \\
4 & 0 & $0 \cdot 35-0 \cdot 45$ \\
5 & $0 \cdot 25-0 \cdot 30$ & $0 \cdot 50-0 \cdot 70$ \\
6 & 0 & $0 \cdot 20-0 \cdot 40$ \\
7 & 0 & $0 \cdot 30-0 \cdot 45$ \\
8 & 0 & $0 \cdot 30-0 \cdot 50$ \\
9 & $0 \cdot 20-0 \cdot 25$ & $0 \cdot 50-0 \cdot 70$ \\
10 & $0 \cdot 25-0 \cdot 30$ & $0 \cdot 45-0 \cdot 55$ \\
11 & 0 & $0 \cdot 30-0 \cdot 35$ \\
12 & 0 & $0 \cdot 15-0 \cdot 35$ \\
13 & $0 \cdot 25-0.30$ & $0 \cdot 40-0 \cdot 45$ \\
14 & 0 & $0 \cdot 25-0 \cdot 40$ \\
15 & 0 & $0 \cdot 30-0 \cdot 35$ \\
16 & $0 \cdot 18-0 \cdot 25$ & $0 \cdot 30-0 \cdot 40$ \\
& & \\
\hline
\end{tabular}

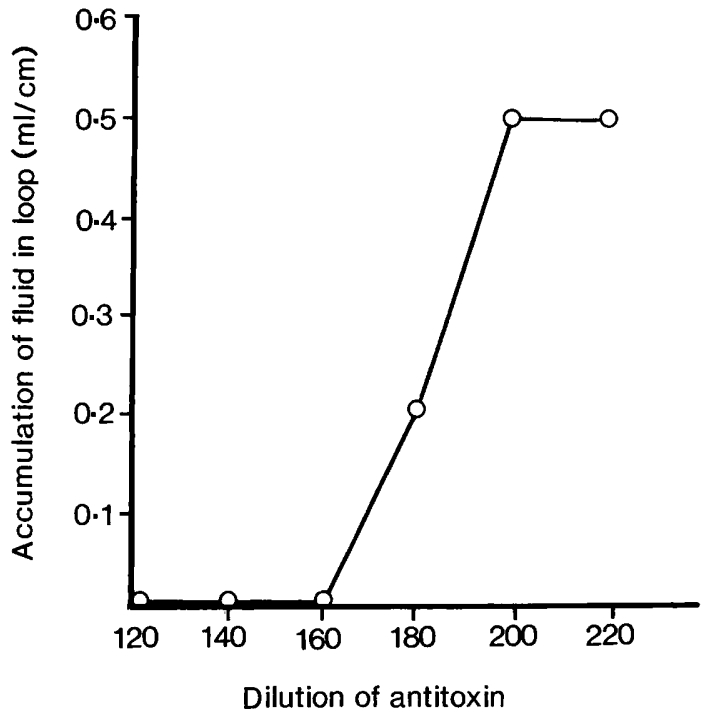

Fig. 2. Neutralisation of $C$. jejuni enterotoxin by cholera antitoxin. The results are the means for filtrates of five cultures.

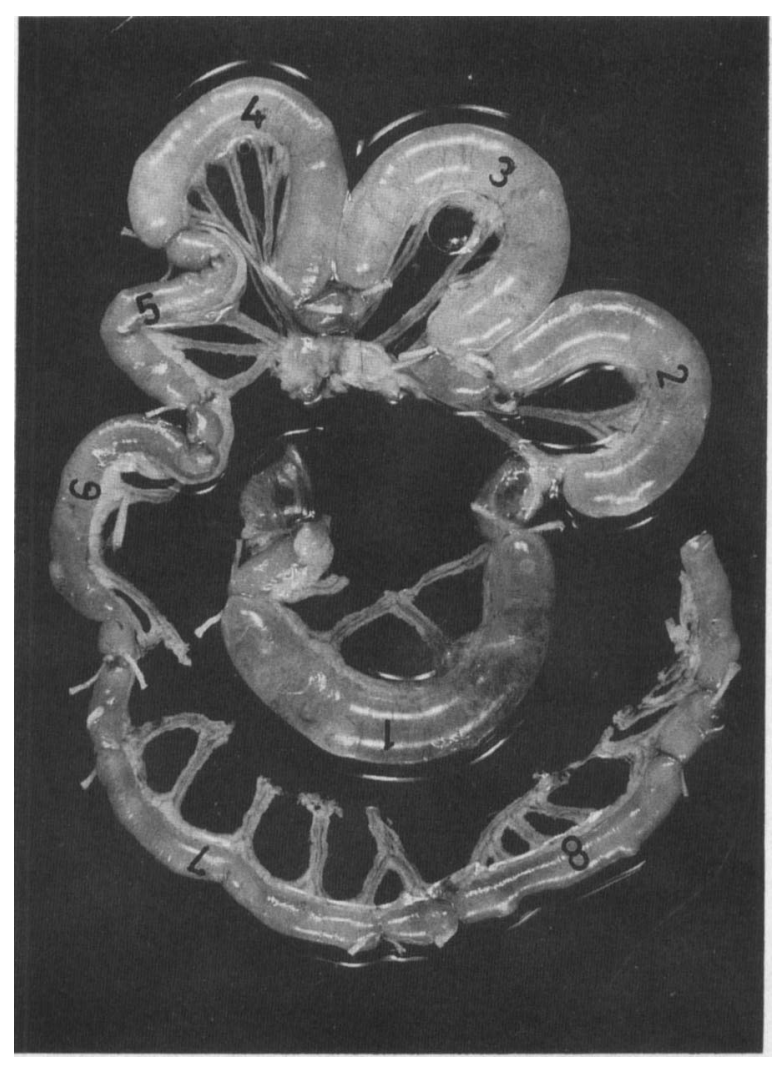

Fig. 3. Neutralisation of $C$. jejuni enterotoxin by cholera antitoxin. Loop 1: positive control, $0.25 \mathrm{ml}$ of culture filtrate mixed with $0.25 \mathrm{ml}$ of PBS. Loops 2-7:0.25 ml of culture filtrate mixed with $0.25 \mathrm{ml}$ of 1 in 220,1 in 200,1 in 180,1 in 160,1 in 140 and 1 in 120 dilutions of cholera antitoxin respectively. Loop 8 : negative control, $0.5 \mathrm{ml}$ of Brucella broth. 


\section{Discussion}

The observation that New Zealand albino rabbits were not susceptible to either live cells or culture filtrates of the chicken isolates of $C$. jejuni is difficult to explain. Our earlier studies with diarrhoeal isolates of $C$. jejuni from children yielded similar results (unpublished data). The explanation put forward by Ruiz-Palacios et al. (1983) that rabbits lack receptors for $C$. jejuni toxin seems untenable in the light of a later experiment by Klipstein and Engert (1985) in which the B sub-unit of $C$. jejuni toxin reacted with $\mathrm{GM}_{1}$ ganglioside in an enzymelinked immunosorbent assay. Age, weight or breed of the animal may, however, play an important role.

Ruiz-Palacios et al. (1983) first reported that some, but not all human diarrhoeal isolates of $C$. jejuni caused accumulation of fluid $(0.28 \pm 0.05 \mathrm{ml} /$ $\mathrm{cm}$ of gut) in ligated rat ileal loops. However, no details of the weight and age of the rats, the length and number of loops per animal, and the size of the inocula were given. Walker et al. (1986) concluded that no satisfactory experimental model was available for $C$. jejuni. The present study showed that in Charles-Foster rats weighing $200-250 \mathrm{~g}$ and aged 7-8 months, at least eight gut loops could be made, and that these were consistently susceptible to $C$. jejuni organisms and culture filtrates, both of which stimulated fluid production $(0.2-0.7 \mathrm{ml} / \mathrm{cm}$ of rat gut). Of several media examined for enterotoxin production, the best was Brucella broth, $p \mathrm{H} 6.7$, without additives.

All of 16 chicken strains of $C$. jejuni were enterotoxigenic, and some, though not all, caused fluid accumulation comparable to that caused by the toxigenic strain 569B of $V$. cholerae. Strain-tostrain and loop-to-loop variation in fluid production can be due to strain variation in the amount of toxin released and to biological variations (Shankar et al., 1982). C. jejuni strain 9 was exceptional in

\section{REFERENCES}

Agarwal R K, Sanyal S C 1981 Experimental studies on enteropathogenicity and pathogenesis of group ' $F$ ' Vibrio infections. Zentralblatt für Bakteriologie, Mikrobiologie und Hygiene A. 249: 392-399.

Annapurna E, Sanyal S C 1977 Enterotoxicity of Aeromonas hydrophila. Journal of Medical Microbiology 10 : 317-323.

Blaser M J, Berkowitz I D, La Force F M, Cravens J, Reller L B, Wang W L L 1979 Campylobacter enteritis. Clinical and epidemiological features. Annals of Internal Medicine 91 : 179-185.

Dubey R S, Sanyal S C 1978 Enterotoxicity of Aeromonas hydrophila: skin responses and in vivo neutralisation. that after passage it invariably caused outpouring of fluid at least as great as that caused by the highly toxigenic strain 569B of $V$. cholerae.

Eleven of 16 strains produced little or no fluid until passaged 1-3 times in the gut of rats. Fluid production increased after each passage. The effects of passage on enterotoxigenicity have been observed with $V$. cholerae non $\mathrm{O} 1$ (Singh and Sanyal, 1978; Shankar et al., 1982), Aeromonas hydrophila (Annapurna and Sanyal, 1977), Plesiomonas shigelloides (Sanyal et al., 1980), V. fluvialis (Agarwal and Sanyal, 1981), V. mimicus (Sanyal et al., 1983), CTgene negative $V$. cholerae O1 (Sanyal et al., 1984) and Klebsiella pneumoniae (Raychaudhury et al., 1984). Possibly passage assists the expression of a repressed toxin gene (Singh and Sanyal, 1978). Earlier reports dealing with human diarrhoeal isolates of $C$. jejuni indicated that some or all were non-toxigenic (Ruiz-Palacios et al., 1983; Wadstrom et al., 1983). The present study suggests that all $C$. jejuni strains are potentially enterotoxigenic. The importance of domestic pets (Walker et al., 1986) and chickens (Harris et al., 1986) in human infections with $C$. jejuni is recognised. In developing countries close association between man and animals may lead to a cycle of infection that increases toxigenicity. A similar enhancement of other virulence factors of $C$. jejuni has been suggested by Newell et al. (1985).

Antisera to cholera toxin or E. coli LT inhibit the cytotonic effects of diarrhoeal isolates of $C$. jejuni (Klipstein and Engert, 1984; McCardell et al., 1984). The present study shows that the enterotoxin produced by chicken isolates can be completely neutralised by cholera antitoxin diluted 1 in 160 , indicating the close immunobiological similarity of the two toxins.

We are grateful to Ms Setarunnahar Saha and Dr R. K. Agarwal for their constant help. The study was supported in part by the Indian Council of Medical Research.
Zentrablatt für Bakteriologie, Parasitenkunde, Infektionskrankheiten und Hygiene A. 242: 487-499.

George H A, Hoffman P S, Smibert R M, Kreig N R 1978 Improved media for growth and aerotolerance of Campylobacter fetus. Journal of Clinical Microbiology 8: 36-41.

Harris N V, Weiss N S and Nolan C M 1986 The role of poultry and meats in the etiology of Campylobacter jejuni/coli enteritis. American Journal of Public Health 76: 407-411.

Johnson W M, Lior H 1984 Toxins produced by Campylobacter jejuni and Campylobacter coli. Lancet 1: 229-230.

Klipstein F A, Engert R F 1984 Purification of Campylobacter jejuni enterotoxin. Lancet 1 : 1123-1124.

Klipstein F A, Engert R F 1985 Immunological relationship of the B subunits of Campylobacter jejuni and Escherichia coli 
heat-labile enterotoxins. Infection and Immunity 48: 629633.

Lahiri A, Agarwal R K, Sanyal S C 1982 Biological similarity of enterotoxins of Vibrio cholerae serotypes other than type I to cholera toxin and Escherichia coli heat-labile enterotoxin. Journal of Medical Microbiology 15: 429-440.

McCardell B A, Madden J M, Lee E C 1984 Production of cholera-like toxin by Campylobacter jejuni/coli. Lancet 1 : 448-449.

Newell D G, McBride H, Saunders F, Dehele Y, Pearson A D 1985 The virulence of clinical and environmental isolates of Campylobacter jejuni. Journal of Hygiene 94: 45-54.

Raychaudhury A, Agarwal R K, Sanyal S C 1984 Enteropathogenicity of Klebsiella pneumoniae strains isolated from stools of diarrhoeal patients and other clinical specimens: An experimental study. Zentralblatt für Bakteriologie Mikrobiologie und Hygiene A 258: 94-103.

Ruiz-Palacios G M, Torres J, Torres N I, Escamilla E, RuizPalacios B, Tamayo J 1983 Cholera-like enterotoxin produced by Campylobacter jejuni. Characterisation and clinical significance. Lancet 2: 250-252.

Sanyal S C, Huq M I, Neogi P K B, Alam K, Rahman A S M H 1983 Vibrio mimicus as an etiologic agent of diarrhoea and its pathogenesis. Indian Journal of Medical Microbiology 1 : $1-12$.

Sanyal S C, Neogi P K B, Alam K, Huq M I, Al-Mahmud K A 1984 A new enterotoxin produced by Vibrio cholerae $\mathrm{Ol}$. Journal of Diarrhoeal Disease Research 2: 3-12.
Sanyal S C, Saraswati B, Sharma P 1980 Enteropathogenicity of Plesiomonas shigelloides. Journal of Medical Microbiology 13: 401-409.

Shankar P, Agarwal R K, Sanyal S C 1982 Experimental studies on enteropathogenicity of Vibrio cholerae serotypes other than 1. Zentralblatt für Bakteriologie Mikrobiologie und Hygiene A 252: 514-524.

Singh S J, Sanyal S C 1978 Enterotoxicity of the so-called NAG Vibrios. Annales de la Societe Belge de Medicine Tropicale 58: 133-140.

Skirrow M B, Benjamin J 1980 Differentiation of enteropathogenic Campylobacter. Journal of Clinical Pathology 33: 1122.

Smibert R M 1978 The genus Campylobacter. Annual Review of Microbiology 32: 673-709.

Speelman P, Streulens M J, Sanyal S C, Glass R I 1983 Detection of Campylobacter jejuni and other potential pathogens in travellers' diarrhoea in Bangladesh. In: Travellers' diarrhoea : new insights. Scandinavian Journal of Gastroenterology 18 (Suppl. 84): 19-23.

Wadstrom T, Baloda S B, Krovacek K, Faris A, Bengtson S, Walder M 1983 Swedish isolates of Campylobacter jejuni/ coli do not produce cytotonic or cytotoxic enterotoxins. Lancet 2: 911.

Walker R I, Caldwell M B, Lee E C, Guerry P, Trust T J, RuizPalacios G M 1986 Pathophysiology of Campylobacter enteritis. Microbiological Reviews 50: 81-94. 
